\title{
Performance Analysis of Handoff Calls for Quality of Service using Call Admission Control Algorithm for Data, Video and Voice Traffic
}

\author{
Ratnesh Konte \\ M.TECH* (DC) \\ SSSIST, Sehore, M.P., India
}

\author{
Jaikaran Singh \\ Department of Electronics and \\ Communication, \\ SSSIST, Sehore, M.P. India
}

\author{
Neeraj Kumar \\ Department of Electronics and \\ Communication, \\ SSSIST, Sehore, M.P. India \\ Mukesh Tiwari \\ Department of Electronics and \\ Communication, \\ SSSIST, Sehore, M.P. India
}

\begin{abstract}
Call admission control is a very important measure in CDMA system to guarantee the quality of the communicating links. In future wireless networks multimedia traffic will have different QoS requirements. The call admission control is very important measure in CDMA system to guarantee the quality of the communicating link In future wireless networks multimedia traffic will have different QoS requirements. We have proposed call admission and call control algorithms for QoS provisioning for multimedia traffic in a cellular mobile network. In this paper we propose a call admission control algorithm that keeps the dropping probability below a specified level while achieving high channel utilization.
\end{abstract}

\section{Keywords}

3G, Call admission control, Quality of service.

\section{INTRODUCTION}

The first generation and second generation system are offered better voice quality and efficient spectrum utilization through digital modulation techniques but it is not efficient for the better service performances of the selected network traffic. The third generation (3G) systems focused itself primarily on universality, high data rate, flexibility, service quality. The data rate to be supported were at least $144 \mathrm{kbps}$ in vehicular, $394 \mathrm{kbps}$ in outdoor-to-indoor, and up to $2 \mathrm{Mbps}$ in indoor. 3G systems allowed both circuit switching and packet switching and opened the possibility to provide advanced and flexible quality of service (QoS) support. Delay sensitive Services, such as voice and video, were served in circuitswitched mode. while data traffic, which can tolerate relatively long delay, have been through packet switched mode to efficiently utilize the system resource.

\section{CALL ADMISSION CONTROL IN MOBILE WIRELESS NETWORKS}

Call Admission Control (CAC) is defined as a system for managing arriving traffic, at the call, connection or session level based on some predefined criteria. A CAC scheme admits, rejects or delays the incoming calls based on a criteria to achieve some QoS objectives for new and existing users [3]. The performance requirements posed by users are measured in terms of quality of service (QoS) and grade of service (GoS). GoS is a call-level factor, which includes new call blocking probability and handoff call blocking probability. Call Admission Control (CAC) ensures network integrity by restricting access to the network so as to avoid overload and congestion, and ensures that QoS requirements of all ongoing connections are satisfied. Resource provision is the major requirement to provide integrated services through wireless mobile communication systems such as voice, data, images, paging, e-mail and multimedia services. Our CAC algorithm reduces the congestion, call blocking and call dropping by creating a limited number of call connections in the network. CAC algorithms achieve efficient resource management by limiting the number of admitted calls and by ensuring QoS to users. Since mobile users experience severe service disruption when handoff calls are dropped, many CAC algorithms employ guard channels for prioritizing and protecting handoff calls [1].

\section{ALGORITHM}

In a mobile wireless cell, the base station has limited power resources. In other words the combined transmitted power to all mobile terminals cannot exceed a certain maximum level. Each newly admitted call makes this resource further scarce. Hence it is very important to adopt policy that will determine which calls to efficiently admit, block or reject based on available resources. Call admission control algorithm ensure that the admission of new call into the resource constrained network does not violate the ongoing users. The following outlines some of the basic needs for which CAC is employed.

- Maximize revenue.

- Fair resource sharing.

- Guarantee transmission rate.

- Guarantee QoS at packet level.

- Some UMTS classes given priority over others.

- Guarantee call-dropping probability.

- Guarantee signal quality. 


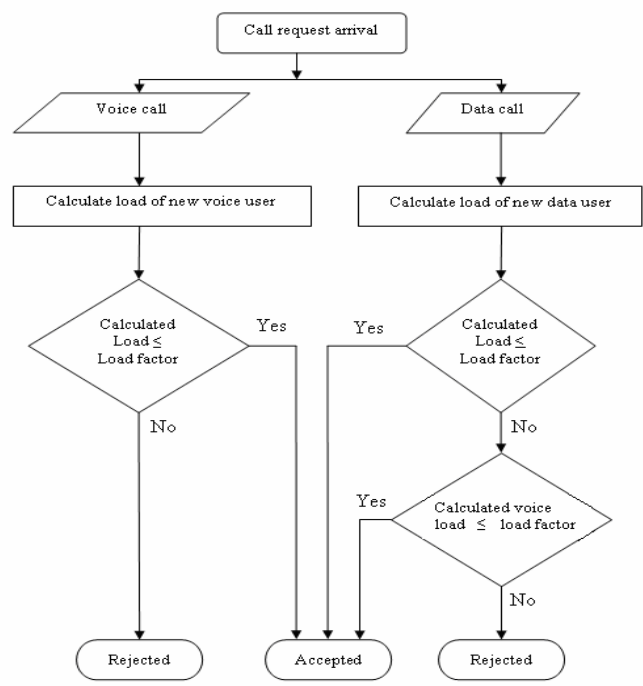

Fig.1. Flow chart of CAC scheme

Our algorithm differs from those algorithms in terms of using the cell load as an admission criteria and also using queuing as an additional priority techniques for handoff calls. Also, the handoff calls is divided into two classes (voice and data), each has its own QoS requirements.

\section{QUALITY OF SERVICE}

Quality of service represents the better service performance for the selected network traffic. It work on priority including dedicated bandwidth, controlled jitter and latency (required by some real-time and interactive traffic), and improved loss characteristics without disturbing the other traffic. The call blocking probability, defined as a probability that new call request is denied service by the network and is subjected to relevant decision made by the CAC scheme employed.

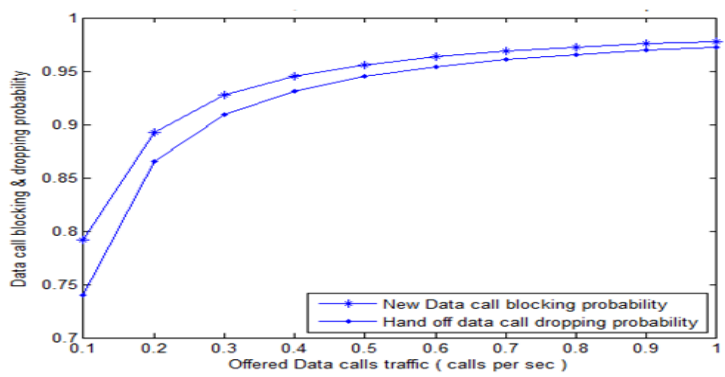

Fig: 2 Data traffic new and hand off calls are treated equally and the hands off calls are allowed to be queued.

New calls and handoff calls are treated equally. and in addition to that, the hand off calls are allowed to be queued. Handoff calls are given higher priority to new calls, and load factor threshold for handoff calls and new calls are different.

$G o S j=\alpha * P h j+P n j$

Here only first three points consider for calculating grade of service

1) Handoff blocking probability calls belonging to traffic of type $\mathrm{j}=0.74$, and

New call blocking probability of calls belonging to traffic of type $\mathrm{j}=0.79$

In this $\alpha=10$, then $\mathrm{GoS}=10 * 0.74+0.79=8.19$
2) Handoff blocking probability calls belonging to traffic of type $\mathrm{j}=0.86$, and

New call blocking probability of calls belonging to traffic of type $\mathrm{j}=0.89$

In this $\alpha=10$, then $\mathrm{GoS}=10 * 0.86+0.89=9.49$

3) Handoff blocking probability calls belonging to traffic of type $\mathrm{j}=0.9$, and

New call blocking probability of calls belonging to traffic of type $\mathrm{j}=0.93$

In this $\alpha=10$, then $\mathrm{GoS}=10 * 0.9+0.93=9.93$

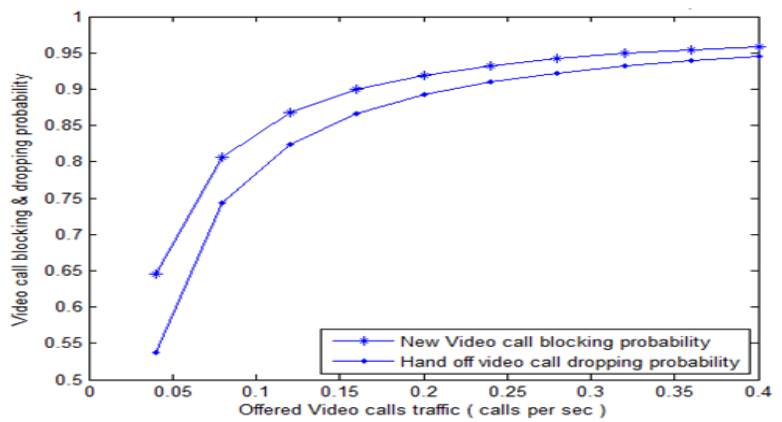

Fig: 3 Video traffic new and hand off calls are treated equally and the hand off calls is allowed to be queued.

New calls and handoff calls are treated equally and in addition to that, the hand off calls are allowed to be queued. Handoff calls are given higher priority to new calls, and load factor threshold for handoff calls and new calls are different. $G o S j=\alpha * P h j+P n j$

Here only three points consider for calculating grade of service

1) Handoff blocking probability calls belonging to traffic of type $\mathrm{j}=0.54$, and

New call blocking probability of calls belonging to traffic of type $\mathrm{j}=0.64$

In this $\alpha=10$, then $\mathrm{GoS}=10 * 0.54+0.64=6.04$

2) Handoff blocking probability calls belonging to traffic of type $\mathrm{j}=0.74$, and

New call blocking probability of calls belonging to traffic of type $\mathrm{j}=0.8$

In this $\alpha=10$, then $\mathrm{GoS}=10 * 0.74+0.8=8.2$

3) Handoff blocking probability calls belonging to traffic of type $\mathrm{j}=0.81$, and

New call blocking probability of calls belonging to traffic of type $\mathrm{j}=0.84$

In this $\alpha=10$, then $\mathrm{GoS}=10 * 0.81+0.84=8.94$

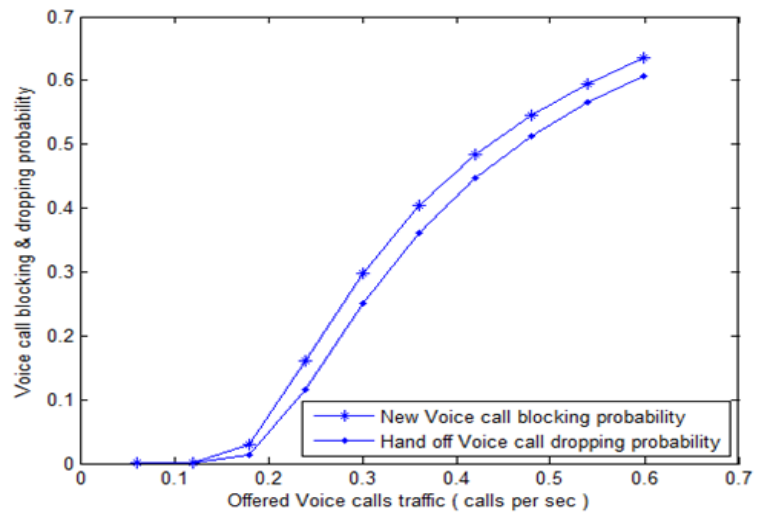

Fig: 3 Voice traffic new and hand off calls are treated equally and the hand off calls is allowed to be queued. 
New calls and handoff calls are treated equally. and in addition to that, the hand off calls is allowed to be queued.

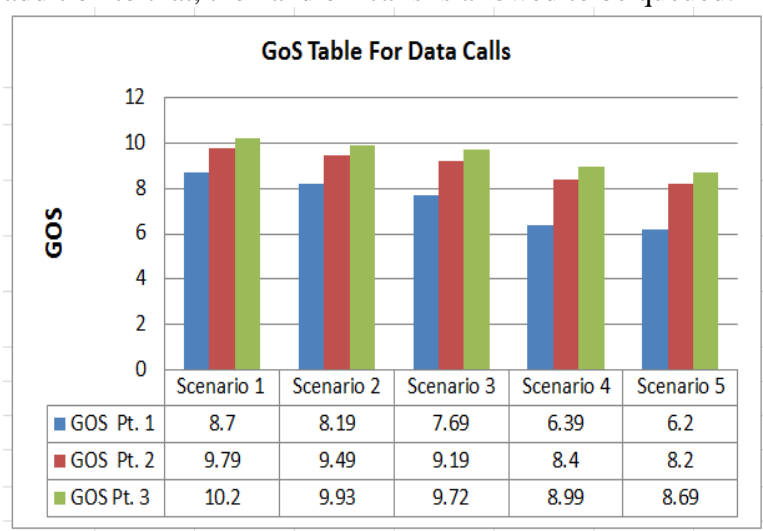

Fig: 4 Grade of service for data call.

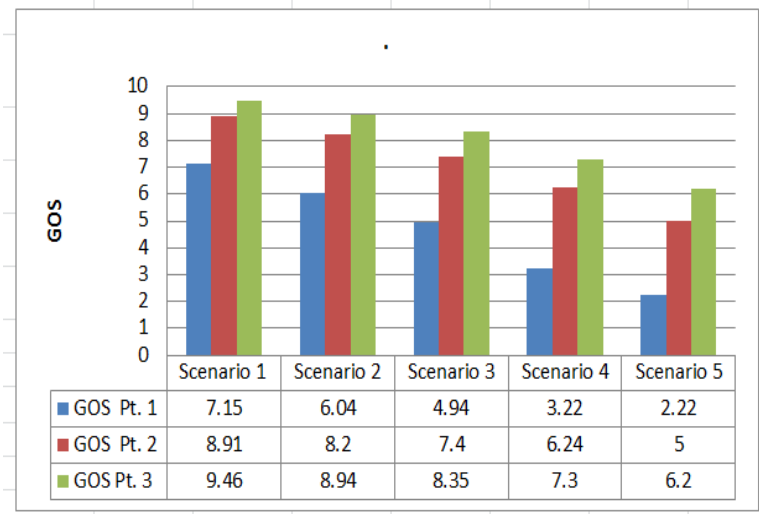

Fig:5 Grade of service for Video call

Handoff calls are given higher priority to new calls, and load factor threshold for handoff calls and new calls are different. $G o S j=\alpha * P h j+P n j$

Here only three points consider for calculating grade of service

1) Handoff blocking probability calls belonging to traffic of type $\mathrm{j}=0$, and

New call blocking probability of calls belonging to traffic of type $\mathrm{j}=0$

In this $\alpha=10$, then $\operatorname{GoS}=10 * 0+0=0$

2) Handoff blocking probability calls belonging to traffic of type $\mathrm{j}=0$, and

New call blocking probability of calls belonging to traffic of type $\mathrm{j}=0$.

In this $\alpha=10$, then $\mathrm{GoS}=10 * 0+0=0$

3) Handoff blocking probability calls belonging to traffic of type $\mathrm{j}=0.02$, and

New call blocking probability of calls belonging to traffic of type $\mathrm{j}=0.04$

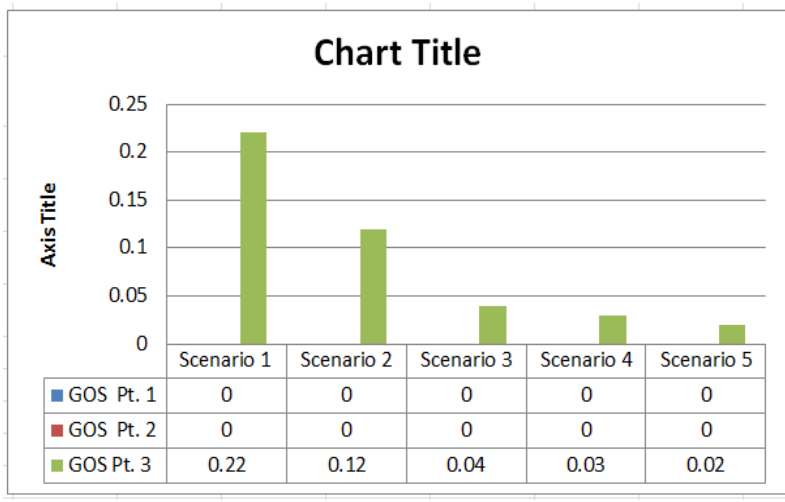

Fig: 6 Grade of service for Voice call

From this graphical analysis at five points, it is clear that the performance get better as use the queue and the soft protector channel. Quality of service improves with decrease in grade of service. Also, as the channel holding time reduces the system performance increase. So as the service time decreases the waiting calls will have better chance to get the channel before they timed out. It is clear that our proposed algorithm has improved system capability and this enhancement raise as channel holding time reduce.

\section{CONCLUSION}

In this paper, the uplink capacity and load estimation formulas is formulated. Then, a prioritized throughput based uplink call admission control algorithm for a WCDMA cellular system with perfect power control is presented. To give priority to soft handoff calls, we introduce queuing techniques and the idea of 'soft guard channels', which is represented by reserving a small fraction of the cell load for the higher priority calls. The performance of this admission control with different scenarios is studied. Based on simulation results, we conclude that this algorithm reduces the dropped soft handoff calls and improves the overall system capacity.

\section{REFERENCES}

[1] Ratnesh konthe,Neeraj Kumar, Jaikaran singh" Call Admission Control for QoS and Performance Analysis of Handoff calls" International Journal of Engineering Trends and Technology (IJETT) - Volume 9 Number 2 Mar 2014

[2] Syed M. Qasim, Shuja A. Abbasi, "Throughput Latency Implementation of Matrix Multiplication using Field Programmable Gate Array" IEEE Transaction on Very Large Scale Integration (VLSI) Systems, vol. 26, no.6, Nov. 2012

[3] Shu-Qing Li, Chi Hou Chan, Leung Tsan "Parallel Implementation of the Sparse- Matrix/Canonical Grid Method for the Analysis of Two-Dimensional Random Rough Surfaces (Three-Dimensional Scattering Problem) on a Beowulf System "IEEE Transaction on Geo Science and Remote Sensing, vol. 38, no. 4, July 2000.

[4] Davide Anastasia and Yiannus Andreopoulos, "Throughput-Distortion Computation Generaic Matrix Multiplication: Toward A Computation Channel for Digital Signal Processing System" IEEE Transaction on Signal Processing, vol.60, no.4, April 2012.

[5] Nan Zhang "A Novel Parallel Scan for Multicore Processors and Its Application in Sparse Matrix-Vector 
Multiplication" IEEE Transaction on Parallel and Distributed System, vol. 23, on. 3, March 2012.

[6] Bahram Hamraz, Nicholas HM Caldwell, and P. John Clarkson "A Matrix-Calculation-Based Algorithm for Numerical Change Propagation Analysis" IEEE Transaction on Engineering Management, vol. 60, no. 1, February 2013.
[7] Vasileios Karakasis, Theodoros Gkountouvas, Kornilios Kourtis, Georgios Goumas, Nectarios Koziris "An Extended Compression Format for the Optimization of Sparse Matrix-Vector Multiplication" IEEE Transaction on Parallel and Distributed System- 2012. 